\title{
Autogestión y asistencialismo: dos extremos del debate sobre el acceso a internet en zonas rurales colombianas
}

\author{
Self-management and welfare: two extremes of the debate on internet \\ access in Colombian rural areas
}

Patricia Martínez Coral ${ }^{*}$

Rec: 9/10/2019

Acep: $3 / 12 / 2019$

\section{Resumen}

Motivado por la creciente influencia de los modelos de desarrollo basados en la comunidad, el presente artículo se propone responder el interrogante sobre la viabilidad de encontrar en el cooperativismo, una alternativa para resolver las necesidades de acceso a internet en las zonas rurales del país. Para ello, se condujo una investigación no-experimental mediante un método mixto, en el que se combinaron recursos cuantitativos y cualitativos, procesados con fundamento en entrevistas estructuradas, dirigidas a través de un muestreo aleatorio a los administradores de las soluciones de acceso comunitario a internet provistas a nivel nacional por el Fondo de Tecnologías de la Información y las Comunicaciones, y un ejercicio de triangulación con fuentes complementarias de información acerca de la calidad de vida en el campo. Los resultados sugieren que en Colombia las problemáticas de exclusión han deteriorado el tejido social de la población rural, lo cual ha obstaculizado el cooperativismo; la apropiación tecnológica no ha alcanzado un grado de maduración tal que le permita convertirse en una necesidad de consumo común, y los esfuerzos realizados por el Estado para fomentar la economía solidaria -especialmente en la ruralidad-, han sido insuficientes. Por ende, se concluye que estos referentes de desarrollo resultan ajenos a las características socio-económicas, políticas y culturales del entorno rural colombiano.

Palabras clave: cooperativismo, desarrollo, acceso a internet, ruralidad.

* Ph.D en Estudios Políticos, magíster en Gobierno y Políticas Públicas, profesional en Gobierno y Relaciones Internacionales. Investigador posdoctoral de la Universidad Externado de Colombia. Correo electrónico: patricia.martinez@est.vexternado.edu.co; Orcid: https://orcid.org/00000002-3787-9827 


\section{Abstract}

Motivated by the growing influence of community-based development models, this article intends to address the question of the feasibility of finding in cooperativism, an alternative to meet the needs of internet access in rural areas. To this end, a non-experimental investigation was conducted using a mixed method, combining quantitative and qualitative resources, based on structured interviews, directed through a random sampling to the administrators of public internet access solutions, provided at the national level by the Information and Communication Technologies Fund, and a triangulation with complementary sources of information about the quality of life in rural areas. The results suggest that in Colombia, exclusion problems have deteriorated the social fabric of rural population, hindering cooperativism; technological adoption has not reached a degree of maturity so that it can become a need for common consumption; the efforts made by the State to promote a solidarity-based economy, especially in rural areas, have been insufficient. Therefore, it is concluded that these referents of development are unfamiliar to the socio-economic characteristics, political and cultural characteristics of the Colombian rural environment.

Keywords: cooperativism, development, internet access, rurality.

\section{Introducción}

El desarrollo basado en la comunidad representa una aproximación connotada del pensamiento económico contemporáneo, enmarcado en la búsqueda de alternativas para la superación de la pobreza. Este enfoque, además de cautivar a organismos multilaterales comprometidos con la adopción de fórmulas eficientes para promover el desarrollo socioeconómico, encuentra eco en altas esferas de la tecnocracia, por proponer una visión que trasciende la dicotomía entre intervención estatal y mercado.
Durante las dos últimas décadas, los estudios de desarrollo socioeconómico destacan la relevancia del enfoque del desarrollo basado en la comunidad. De acuerdo con el Banco Mundial, el nuevo milenio exige una reformulación de la perspectiva empleada para reducir la pobreza, considera que la literatura económica logró demostrar en la década de 1990 que la separación entre eficiencia y equidad, o bien entre crecimiento y distribución, no es en modo alguno legítima, así como las prescripciones universales del desarrollo deben dar paso a adaptaciones de carácter local. A la luz de estas reflexiones, la dicotomía entre Estado y mercado también se debe superar.

Fue así como este organismo multilateral adoptó los modelos participativos entre las premisas del nuevo marco de referencia para abordar la promoción del desarrollo; de esta manera, involucrar a las comunidades en la toma de decisiones en los proyectos de inversión conduciría a iniciativas mejor estructuradas, con criterios de focalización más precisos, una implementación más transparente, resultados equitativos y logros en costo-eficiencia y sostenibilidad (Banco Mundial, 2001). En 2012, un balance de la implementación de proyectos del Banco Mundial con este enfoque abarcaba un total de 400 iniciativas, distribuidas en 94 países, con una inversión cercana a $\$ 30$ billones de dólares, lo cual representa entre 5 y $10 \%$ de su portafolio de préstamos (Wong, 2012).

Elinor Ostrom (1994), merecedora del Premio Nobel de Economía en 2009 por su obra sobre la gobernanza económica de los bienes comunes, sintetizó este giro como la necesidad de complementar el crecimiento económico generado por el capital físico, con insumos provistos por el capital humano y el capital social, de tal forma que los frutos de la productividad se pudieran combinar con el impacto del conocimiento, entrenamiento, experiencia laboral, y patrones de interacción sustentados en la reciprocidad y la confianza que facilitan las actividades de coordinación económica. Ostrom empleó como objeto de estudio algunos casos que históricamente habían sido 
efectivos para el manejo de bienes comunes, como los sistemas de riego administrados colectivamente; la autora logró ilustrar cómo el diseño de reglas para el acceso y uso del recurso, mecanismos para garantizar su respectivo cumplimiento, instrumentos para resolver conflictos y la autonomía de este esquema de gestión frente a terceros, incluso autoridades públicas, comprueban que existen diversas fuentes de desarrollo fundamentadas en la cooperación social y que escapan a la ortodoxia económica.

No obstante, advirtió Ostrom, es la coincidencia entre los lazos de la cooperación y el entorno físico y cultural en el que tienen lugar, lo que permite que surja la acción colectiva, evolucione, y sobreviva con el paso del tiempo. Esta observación reviste especial importancia en la medida que los beneficios del comportamiento pro-social, encauzado por los proyectos comunitarios, pueden tardar más tiempo del esperado, tal como ocurre en entornos culturales en los que el carácter centralizado de las políticas y algunos valores sociales, inhiben los modelos participativos (Nguyen y Rieger, 2014).

Asimismo, existen diferencias conceptuales acerca de las características que definen a una comunidad, cuya incidencia resulta sustancial para la formulación y evaluación de los proyectos cooperativos. Para la ciencia económica, la acción colectiva se fundamenta en la elección racional, por lo que se asume que las interacciones entre individuos son estratégicas. Desde esta óptica, la estructura de los incentivos que llevan a la cooperación son los aspectos preponderantes para orientar y valorar los proyectos. Desde una perspectiva sociológica, el capital social que explica el comportamiento cooperativo surge de normas, patrones de cohesión, y vínculos comunitarios, por lo que la confianza y la densidad de las redes sociales gozan de especial énfasis en el diseño de las iniciativas.

De otro lado, la psicología social proporciona otra aproximación denominada dinámica intergrupal, según la cual las comunidades se definen a partir de los límites impuestos por las condiciones de membrecía y la forma en que, colectivamente, actúan con otros actores. Desde este ángulo, las categorías identitarias, los tamaños de las agrupaciones y las relaciones simbólicas y materiales de interacción social son variables determinantes para los modelos de desarrollo cooperativo (Bennett y D'Onofrio, 2014).

Como complemento a las diferencias conceptuales provistas por cada enfoque disciplinar, Fraser (2005) identifica cuatro modalidades empleadas en la literatura sobre el desarrollo cooperativo para referirse a las comunidades, con base en los propósitos que les congrega, matices que se desprenden del hecho de que las comunidades marcan un límite difuso entre los dominios público y privado. La primera, correspondiente al conservadurismo económico, atribuye a las comunidades la búsqueda de ganancia, propiedad y espacios de auto-gestión tendientes a disminuir la regulación pública; la segunda es una visión funcionalista que entiende las comunidades como árbitros plurales y objetivos para dirimir conflictos de interés; la tercera es de corte progresivo y toma a las comunidades como espacios participativos, incluyentes y democráticos para la solución de problemas y gestión de recursos compartidos. La última es una modalidad radical que concibe las comunidades como instrumentos de activismo para la transformación del orden socio<económico y/o político.

Además de la falta de consenso en el abordaje de las comunidades, también existen diferencias metodológicas ostensibles entre los estudios del desarrollo cooperativo, tales como la naturaleza de los datos estudiados, el rol del investigador respecto de su objeto de estudio (objetividad y subjetividad), los criterios para validar explicaciones satisfactorias, y el énfasis dado a los procesos de la cooperación o, por el contrario, a sus resultados (Bardhan y Ray, 2008). Es así como el abordaje interdisciplinar puede enriquecer los estudios sobre el desarrollo basado en la comunidad, pero para ello es necesario reconocer las divergencias expuestas y no menos importante, 
tener presente que cada supuesto adoptado como cierto en torno al fenómeno de cooperación social, amerita una reflexión preliminar de fondo.

En este contexto, el presente artículo expone los resultados de una investigación de tipo no experimental orientada a responder si en Colombia el cooperativismo ofrece una herramienta efectiva de autogestión comunitaria, con el potencial de promover el despliegue de infraestructura de telecomunicaciones en zonas rurales y contribuir al cierre de la brecha de acceso a internet. Después de introducir los antecedentes y el marco conceptual sobre los modelos asociativos de base comunitaria aplicado a las telecomunicaciones, se detallan las piezas metodológicas aplicadas en el trabajo de investigación, se exponen los hallazgos obtenidos y a manera de cierre se sintetizan, los elementos de análisis que permiten desvirtuar la aplicabilidad del paradigma enunciado.

\section{Antecedentes y marco conceptual aplicado a las telecomunicaciones}

El creciente interés de formuladores y analistas de política pública por los desafíos que plantea la inclusión digital en países caracterizados por bajas tasas de penetración de internet, ha encontrado una ruta concomitante al estudio del cooperativismo, tras la divulgación internacional de casos de éxito en los que el despliegue de redes de infraestructura ha estado apalancado mediante esquemas asociativos de carácter comunitario. Sin duda, este tipo de experiencias alienta la esperanza de resolver las inagotables discusiones entre la necesidad de subsidiar la oferta del servicio de internet, dadas las economías de densidad que inhiben la inversión privada en el campo, y el objetivo de lograr un diseño óptimo del subsidio que evite distorsionar el mercado y mantener los fines redistributivos en condiciones de eficiencia $y$ sostenibilidad financiera (Parsons y Stegeman, 2018).

En 2005, un reporte especial para el Programa de las Naciones Unidas para el Desarrollo ilustró cómo la creación de cooperativas para la oferta de servicios de telecomunicaciones prometía resolver la falta de incentivos a la inversión privada y la ausencia de recursos públicos para garantizar el acceso universal. Con base en las lecciones aprendidas en países como Polonia, India y Perú, el documento señaló que las redes de infraestructura de propiedad comunitaria se convirtieron en un instrumento costo-eficiente para satisfacer las necesidades de la población rural, aunque para ello fue indispensable crear un clima propicio para asumir tales retos.

Entre las medidas adoptadas se encuentran acciones regulatorias tendientes a simplificar los trámites y permisos para la prestación de servicios en áreas específicas con tecnologías eficientes; beneficios para celebrar acuerdos de interconexión; estímulos financieros para apalancar la inversión; y estrategias de fortalecimiento de capacidades para las organizaciones de base (Ó Siochrú y Girard, 2005).

A su vez, el recorrido de las cooperativas del estado de Wisconsin, Estados Unidos, es otro caso emblemático. Según Kluz (2017), cerca de 11 cooperativas de telecomunicaciones ofrecen servicios de voz y datos en las zonas rurales del estado, que concentran aproximadamente 9 \% de la población, agrupan alrededor de 35.000 suscriptores. Gracias a inversiones en redes que abarcan fibra a las premisas (FTTP), comercializan un ancho de banda casi ilimitado y algunas incluyen servicios de fomento a la demanda, como capacitación virtual para el desarrollo de negocios y comercio electrónico. En este modelo, los miembros de la cooperativa son sus propietarios y tienen el control sobre ella, lo que permite operar y distribuir los servicios a costos bajos, así como mantener una perspectiva de inversión a largo plazo, a diferencia de las compañías que requieren prontos retornos de inversión.

Estos resultados denotan una larga trayectoria de organización comunitaria, como respuesta a las brechas de mercado, pero también un constante apoyo 
gubernamental. En 1949 se creó un programa federal de préstamos blandos para facilitar la creación y mantenimiento de sistemas de telefonía rural, que promovieron así el surgimiento de modelos cooperativos. Con el tiempo, el Departamento de Agricultura de los Estado Unidos pasó a administrar estos fondos, y su impacto ha sido notable en el desarrollo de esquemas similares en el sector eléctrico, pues cerca de $42 \%$ de las líneas de distribución eléctrica en dicho país, en la actualidad, son propiedad de cooperativas.

En América Latina por su parte, sobresalen las experiencias del cooperativismo del Cono Sur. De acuerdo con un informe sobre la contribución de los modelos cooperativos para promover el acceso a Internet en zonas rurales, en 2010 Argentina ya contaba con dos federaciones de cooperativas de telecomunicaciones (Fecotel y Fecosur-) que en conjunto integraban a 350 proveedores de servicios y cerca de 2,5 millones de usuarios, cubría alrededor del $8 \%$ de la población nacional (Alderete y Carrasco, 2010).

Según Landriscini (2013), el proceso histórico de fraccionamiento del territorio argentino desde inicios del siglo XX dio origen a la conformación de diversas colonias rurales en las que se desarrollaron modalidades de autoproducción, subsistencia y comercio soportadas en el trabajo asociativo, conocimientos y prácticas que se fueron trasmitiendo de una generación a otra. De esta manera, la economía solidaria se institucionalizó localmente e impulsó la apertura de espacios de co-gestión con entidades gubernamentales, esto evolucionó hacia la creación de mecanismos de protección jurídica y estímulos técnicos y financieros que han resultado de notable importancia para el mantenimiento de dichas prácticas.

Otro caso sobresaliente es el de la comunidad indígena de Оахаса, México, que posee y opera infraestructura de una red local celular, de modo que ofrece el servicio de voz en un área geográfica restringida y gestiona acuerdos con proveedores de internet para el acceso a datos y soporte técnico.
Desde 2014, la legislación mexicana incorporó dos tipos de concesión especial para prestar servicios de radiodifusión y telecomunicaciones en áreas remotas (uno para organizaciones sociales sin ánimo de lucro y otro para comunidades indígenas) y la destinación específica de bandas de frecuencia para propósitos sociales (entre 824-849 MHz y 869-894 $\mathrm{MHz}$ ) que facilitan la prestación de servicios de telecomunicaciones en comunidades con población entre 200 y 3.000 habitantes (Baca, Belli, Huerta y Velasco, 2018).

Estos referentes motivan las pesquisas sobre el fenómeno del cooperativismo en la ruralidad colombiana y su potencial para propiciar alternativas de cambio tecnológico sustentadas en modelos de apalancamiento de infraestructura local, toda vez que las brechas de acceso a internet comportan un inconmensurable costo de oportunidad para la población marginada de la información y el conocimiento que circulan por el ciberespacio. La Unión Internacional de Telecomunicaciones (ITU) señala que, de acuerdo con las métricas de Cisco, globalmente el usuario promedio de internet consume 28.8 gigabytes (GB) al mes, en un amplio rango que oscila entre 98,7 GB en economías desarrolladas (datos por usuario al mes) y 7,2 GB en países en desarrollo (ITU, 2019).

En Colombia, según la Encuesta de Calidad de Vida del DANE (2019), en 2018 el porcentaje de hogares que contaba con acceso a internet fijo en zonas urbanas ascendía a 50,8 \%, mientras que en zonas rurales estaba en $4,3 \%$, situación que torna crítica la disponibilidad de soluciones de acceso público financiados por el Estado, a través del Fondo Único de Tecnologías de la Información y las Comunicaciones. Debido a los consecuentes esfuerzos por garantizar la oferta del servicio de internet en zonas rurales y apartadas, la sostenibilidad de la inversión realizada por dicha entidad ha dado lugar a cambios recientes en el control de los recursos asociados a la política de acceso universal (DNP, 2017).

Así por ejemplo, las bases del Plan Nacional de Desarrollo 2018-2022 consignaron el objetivo de "generar un modelo sostenible para la 
conectividad social en zonas urbanas y, en especial, rurales", y posteriormente la Ley 1978 de 2019 (ley de modernización sectorial) incluyó como parágrafo de la naturaleza y objeto del Fondo Único de Tecnologías de la Información y las Comunicaciones "[...] Aplicar criterios de factibilidad financiera, social, técnica, económica, jurídica, institucional y de sostenibilidad, para justificar las inversiones en planes, programas y proyectos de su competencia". Este enfoque de la política de acceso universal confirma la pertinencia de validar las expectativas en los modelos asociativos de base comunitaria.

\section{Metodología}

Con el fin de abordar el análisis enunciado, se llevó a cabo una secuencia exploratoria mixta (cuantitativa-cualitativa), enmarcada en una investigación de tipo no-experimental. Con base en un muestreo aleatorio efectuado en un universo de 5.648 comunidades rurales, se desarrollaron 500 entrevistas estructuradas efectivas a los administradores de las soluciones de acceso comunitario a internet, provistas a nivel nacional por el Fondo Único de Tecnologías de la Información y las Comunicaciones, mediante el proyecto Kioscos Vive Digital, cuya ejecución se dio durante el periodo 2013-2019. El total de la muestra de administradores se distribuye además entre cuatro ejecutores de la fase II del proyecto Kioscos Vive Digital, con el fin de evitar posibles sesgos.

Los resultados se presentan agrupados por región y el muestreo garantizó una distribución homogénea entre el número de departamentos pertenecientes a cada una de ellas. La región Caribe comprende los departamentos de Atlántico, Magdalena, Sucre, Bolívar, Cesar, Córdoba y La Guajira; la región oriental abarca Norte de Santander, Santander, Arauca, Casanare y Vichada; la región centro integra Tolima, Antioquia, Boyacá, Caldas, Cundinamarca, Huila, Meta, Quindío y Risaralda; la región Pacífico incluye Chocó, Cauca, Valle del Cauca y Nariño; y la región sur concentra
Putumayo, Caquetá, Guaviare, Guainía, Vaupés y Amazonas. A su vez, el análisis de la información recolectada se soportó en un ejercicio de triangulación con fuentes complementarias que amplían el conocimiento acerca de las condiciones que permean el tejido social en la ruralidad colombiana.

Por último, es preciso indicar que la idoneidad de los administradores de las soluciones de acceso comunitario a internet, para servir como foco de las estimaciones acerca de las posibilidades reales de promover esquemas cooperativos para la prestación del servicio en zonas rurales, obedece a los criterios empleados por el Ministerio TIC (MinTIC) para validar su respectiva selección, por parte de los ejecutores del proyecto Kioscos Vive Digital. De acuerdo con los términos del proceso licitatorio para la contratación de los ejecutores del proyecto, el administrador de cada kiosco debía acreditar como mínimo el siguiente perfil:

Ser mayor de diez y ocho (18) años de edad; saber leer y escribir; tener conocimiento básico y práctico de computadores y/o sistemas e Internet [sic], para lo cual deberá aprobar un examen de suficiencia y conocimiento en el uso de computadores e Internet [sic], diseñado por el contratista y validado por la interventoría, de lo cual deberá dar prueba el contratista; demostrar reconocimiento de la comunidad por su liderazgo y vocación hacia el desarrollo comunitario. (Se sugiere validar la información mediante una (1) carta de referencia que dé cuenta de la gestión y capacidad de liderazgo de la persona.); tener disponibilidad para atender el Kiosco Vive Digital mínimo por veinte (20) horas a la semana. (SECOP, 2013)

Asimismo, se corroboró la importancia del rol social que desempeñan estos administradores en las comunidades rurales en las que se ubican las soluciones de acceso comunitario a internet con una evaluación de impacto del proyecto, en la que consta que las actividades desarrolladas en los Kioscos no solo 
han generado efectos positivos en las condiciones de uso y apropiación de la tecnología, sino también en la construcción de tejido social (DNP, 2015).

\section{Resultados}

Después de indagar sobre las percepciones de los administradores de los centros de acceso comunitario a internet acerca de los modelos asociativos como mecanismo para satisfacer las necesidades locales de conectividad, se evidenciaron diversos retos aún por superar. A la pregunta: "¿considera viable que la comunidad rural donde se ubica el kiosco desarrolle una iniciativa propia para proveer el servicio de conectividad a Internet?", en promedio, las opiniones se dividieron en una proporción muy cercana (52 \% considera que es viable, y 47,8 \% que no lo es), la inclinación positiva más alta se dio en la región central (65\%), y la más baja en la región oriental (46\%). No obstante, pese a que muchos administradores estimaron posible el desarrollo de formas asociativas en su comunidad para ofrecer acceso a internet, la gran mayoría manifestó no conocer casos concretos de servicios ofrecidos a través de estos modelos.

A la pregunta: "¿conoce algún caso de servicios provistos en el centro poblado mediante una cooperativa u otro modelo asociativo?", 89,8 \% respondió que no, y solo 10,2 \% que sí. Ahora bien, entre los ejemplos mencionados por los entrevistados, ante la solicitud de ilustrar su respuesta, se citan asociaciones de productos agrícolas, galpones y criaderos de pollos, cooperativas de transporte, abastecimiento de víveres, producción de leche, cultivadores de café y acueductos veredales.

Entre las opciones que los entrevistados consideran más relevantes para explicar las razones por las que no estiman posible que el servicio de internet sea provisto por alguna forma asociativa en la comunidad rural, sin que estas fueran excluyentes entre sí, sobresale la carencia de recursos económicos, seguida de la falta de conocimientos técnicos, la fragilidad de la confianza y otros aspectos de relacionamiento social $y$, en menor grado, los problemas de orden público. La figura 1 ilustra la distribución de percepciones por región.

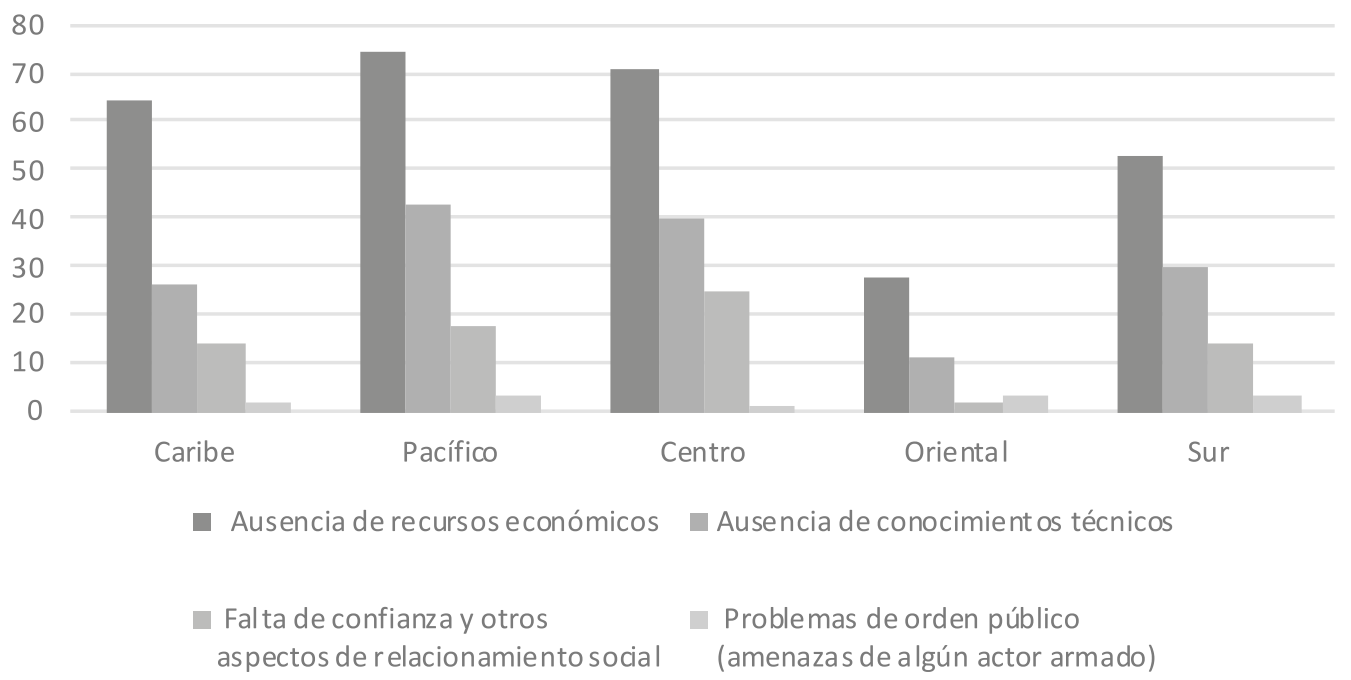

Figura 1. Percepción sobre la viabilidad de desarrollar una iniciativa comunitaria para proveer el servicio de Internet Fuente: elaboración propia 
A su vez, entre las razones complementarias que los entrevistados consideran importantes se encuentran:

la inestabilidad en las actividades que generan ingresos en el campo; las características geográficas que dificultan la instalación del servicio de internet; la falta de liderazgo entre los miembros de la comunidad; el que la gente no tiene cómo pagar las tarifas para hacer uso del servicio; la falta de apoyo y solidaridad en la comunidad; la carencia de interconexión eléctrica; los campesinos se dedican a las actividades agrícolas para subsistir; la comunidad espera recibir los servicios por parte del Estado; la dificultad para lograr acuerdos al interior de la comunidad; para muchas personas en el campo, especialmente para los adultos mayores, internet no es una necesidad.

Las respuestas al interrogante: "si el Ministerio TIC no tiene los recursos para seguir financiando los Kioscos, ¿quién considera que debe ser responsable de hacerlo?", los encuestados evidenciaron de modo contundente, que las formas asociativas no hacen parte del imaginario social. A través de un consenso significativo, el Estado fue reconocido como único proveedor. Solo en la región Pacífico, las respuestas de "Gobernación, Alcaldía, Gobierno Nacional y Ministerio de Educación" fueron complementadas con la categoría de "organizaciones no gubernamentales (ONG)". Con el propósito de precisar si hay diferencias sustanciales en la percepción de los entrevistados sobre las responsabilidades que les asiste a los estamentos gubernamentales y su respectiva voluntad y/o capacidad de asumirlas, se preguntó: "eestima viable que la alcaldía o gobernación que corresponda al centro poblado donde se ubica el kiosco se encargue de financiar el acceso a internet?". Las opiniones se dividieron con un margen estrecho. El agregado nacional respondió afirmativamente en 56,6 \%, siendo la región Pacífico la única con una inclinación por debajo de la media, tal como se ilustra en la figura 2.

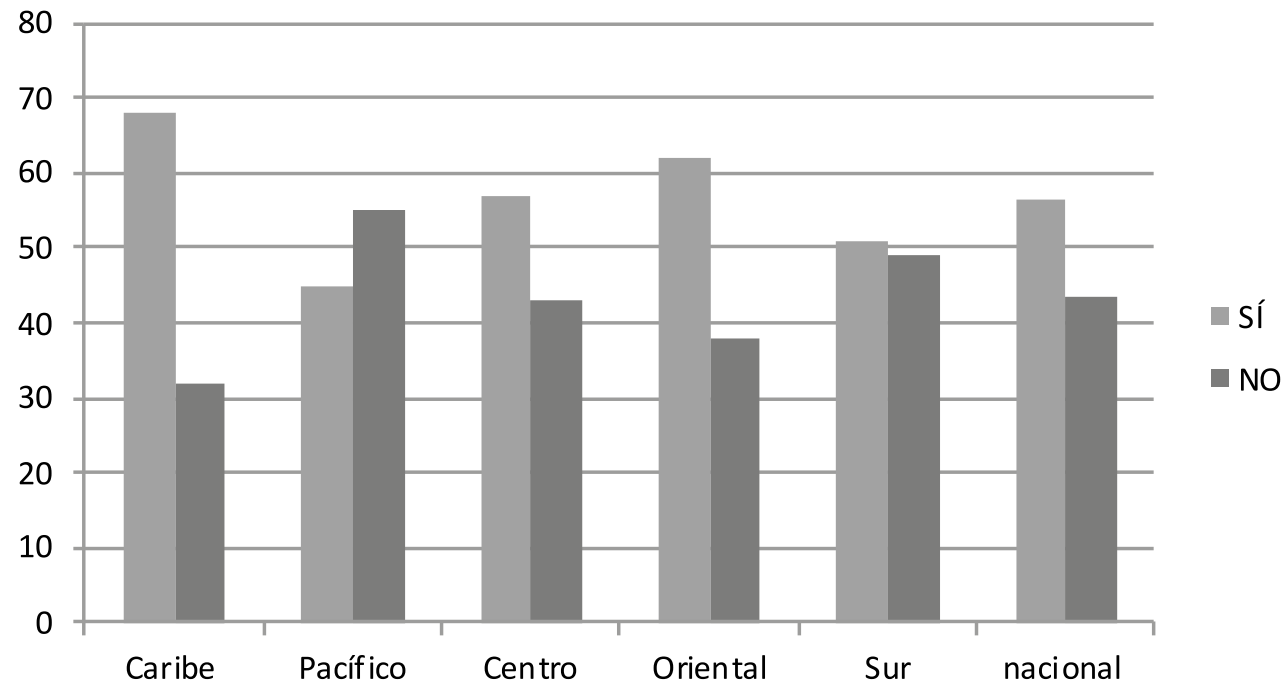

Figura 2. Percepción sobre la viabilidad de que el ente territorial financie el acceso a internet en el centro poblado Fuente: elaboración propia

La mayoría de quienes consideraron viable que el ente territorial financiara el acceso a internet argumentaron que es responsabilidad suya velar por el bienestar de las comunidades y ofrecer medios para el progreso social. Estas afirmaciones permiten comprender que abordan el interrogante a partir de 
supuestos y expectativas que no necesariamente encuentran asidero en la realidad. El referente concreto más representativo para describir esta disonancia se advierte en los indicadores de conectividad escolar, bajo el entendido que los recursos del sistema general de participación que administran los entes territoriales constituyen la principal fuente de financiación de este servicio.

Al cierre de 2018, en promedio, la matrícula conectada a nivel nacional se acercaba a $43 \%$, de la cual $31 \%$ correspondía a sedes ubicadas en zonas urbanas y $12 \%$ a la matrícula rural. La magnitud de esta brecha se aprecia mejor al precisar que de las 43.545 sedes oficiales registradas a nivel nacional, cerca de $80 \%$ se distribuían en zonas rurales, concentrando tan solo $28 \%$ de la matrícula estudiantil, en tanto que el $20 \%$ restante ubicado en zonas urbanas concentraba $72 \%$ de la población escolar (Ministerio de Educación Nacional [MEN], 2019).

De otro lado, entre las razones expuestas por aquellos que respondieron negativamente a la pregunta sobre la viabilidad de financiar el acceso a internet con cargo a los recursos de las autoridades locales se encuentran aseveraciones tales como:

[...] hay mucha corrupción; los únicos proyectos que financian los entes locales son los que dan beneficios políticos; las entidades territoriales no cuentan con suficientes recursos; las entidades locales no se preocupan por las comunidades rurales; los mandatarios locales nunca cumplen sus promesas; los entes territoriales tienen otras prioridades como la salud; a los gobiernos locales no les interesa que la gente del campo se eduque y progrese; si el centro poblado no votó por el alcalde o gobernador no le financian proyectos; en el pasado, los entes territoriales no han invertido en las comunidades rurales.

En suma, el procesamiento de la información obtenida corrobora la precariedad del cooperativismo como base del desarrollo comunitario en las zonas rurales del país, lo que guarda consistencia con otros estudios que han tenido por objeto analizar este tipo de prácticas, así como detallar y comprender las condiciones necesarias para mejorar la calidad de vida en el campo. Un diagnóstico elaborado en 2016 acerca de los concesionarios de radiodifusión sonora, permitió al MinTIC determinar que el servicio de radio comunitaria en zonas rurales del país se caracteriza por un modelo fallido de operación que hace inviable financiar las emisoras bajo esquemas autosostenidos, debido a la falta de conocimientos técnicos y la orientación de los contenidos que integran su parrilla, lo que dificulta fidelizar audiencias.

Del mismo modo, las verificaciones realizadas in situ en las emisoras comunitarias demuestran la inobservancia del régimen aplicable a la radiodifusión sonora, por cuanto no existe alineación entre los contenidos difundidos -que en su mayoría son programas musicales genéricos- y los objetivos consagrados en la normatividad vigente, tales como la generación de espacios de expresión, información, educación, comunicación, promoción cultural, formación, debate y participación comunitaria (MinTIC, 2016).

Por su parte, el informe de la misión para la transformación del campo (Ocampo, 2014) señala que una razón histórica de la fragilidad de los lazos cooperativos en la ruralidad colombiana está dada por el enfoque asistencialista, con base en el cual se han diseñado los instrumentos de acción pública orientados a disminuir la pobreza. Tras un continuo proceso de reestructuración de las instituciones ejecutoras de política rural, los estímulos creados para el desarrollo productivo del campo no han logrado transformar las condiciones más críticas que enfrenta el campesinado; del mismo modo que la descentralización administrativa, política y fiscal consagradas en la Constitución Nacional de 1991, lejos de contribuir al proceso de cambio, han favorecido a pequeños núcleos urbanos, mantuvieron así las brechas de cobertura y calidad de servicios entre el campo y la ciudad. 
Entre las necesidades más apremiantes, este informe menciona la de impulsar un reordenamiento social de la propiedad que permita formalizar títulos, ampliar minifundios, fomentar nuevas modalidades de arrendamiento y consolidar territorios comunales para promover el acceso a los activos productivos. También sugiere facilitar el acceso a servicios financieros mediante esquemas de ahorro, crédito y aseguramiento contra riesgos para fortalecer de manera simultánea la cultura de pago.

El informe de desarrollo humano elaborado por el Programa de las Naciones Unidas para el Desarrollo (PNUD) en 2011 arroja elementos de análisis adicionales para explicar por qué el cooperativismo no ha tenido arraigo en la ruralidad. De un lado, el informe reconstruye el fenómeno de desconocimiento político que ha sufrido el campesinado a lo largo de la historia y cuyo origen se remonta a las estructuras sociales de la época colonial. Esta forma de exclusión le ha impedido a los campesinos consolidar comunidades políticas, y lograr procesos sostenidos de interlocución con el Estado, problemática agudizada por la prominencia de las élites políticas, en virtud de la cual sectores como el campesino han contado, tradicionalmente, con déficits de representación. Asimismo, el informe expone cómo el despojo de tierras del que ha sido víctima la población rural a causa de la violencia, ha erosionado las redes sociales y la unidad familiar, destruye así los proyectos de vida común en el campo (PNUD, 2011).

Al tomar en consideración que las soluciones de acceso comunitario a internet ofrecidas por el Fondo Único de Tecnologías de la Información y las Comunicaciones se localizan en centros poblados rurales (veredas, corregimientos, inspecciones de policía, y caseríos) en los que habitan como mínimo 100 personas, es posible inferir que la población que se distribuye en el área rural dispersa enfrenta problemáticas aún más críticas para impulsar el cooperativismo. El Censo Nacional Agropecuario (CNA) proporciona una descripción amplia sobre las condiciones de vida de dicha población (DANE, 2016). Tras procesar datos de
113.008.623 hectáreas, equivalentes al 99 \% del área rural continental e insular del país, se encontró que: $56,7 \%$ corresponden a bosques naturales, 38,6 \% tienen uso agropecuario, un 2,2\% uso no agropecuario y 2,5\% están asignadas a usos complementarios.

De la cantidad de unidades productoras agropecuarias censadas, el régimen de tenencia de la tierra se declaró mayoritariamente como propio (72,7 \%) con respecto a las demás modalidades (arriendo, aparcería y propiedad colectiva) y solo $16,4 \%$ de estas unidades reportó tener maquinaria para el desarrollo de las actividades agropecuarias. Entre los datos sociodemográficos relevantes del CNA sobresale que del total del área rural dispersa censada, 15,7\% de las viviendas ocupadas no tiene ningún servicio público; 82,9 \% tiene energía eléctrica, 42,5\% cuenta con acueducto, y solo 6 \% con alcantarillado.

El promedio de personas por hogar rural es de 4,23; el mayor nivel educativo alcanzado por 54,1 \% de la población en el área rural dispersa es básica primaria, seguido por "ninguno" con 19,2 \%, y 12,6 \% de la población con 15 años o más es analfabeta; y aunque la afiliación a la seguridad social en salud es superior a $90 \%$, el régimen preponderante es el subsidiado (82,2 \%). Una interpretación global del índice de pobreza multidimensional sugiere que 45,7 \% de la población del área rural dispersa vive en condiciones de pobreza. Adicionalmente, el CNA indagó por las dinámicas de asociatividad e identificó que 73,7 \% de los productores que residen en el área rural dispersa no pertenece a ninguna asociación; 11,9 \% manifestó pertenecer a organizaciones comunitarias como consejos $u$ organizaciones étnicas, de mujeres, ancianos o jóvenes; y solo 1,2 \% reportó pertenecer a alguna agremiación.

Además de las condiciones estructurales que inhiben las formas asociativas como instrumento para la promoción del desarrollo comunitario, las entrevistas realizadas a los administradores de los Kioscos Vive Digital para sondear si tal esquema podría, en principio, resultar aplicable a la oferta de acceso a internet en 
zonas rurales, revelan que la apropiación de la tecnología continúa siendo un reto para agrupar una demanda efectiva de servicios con la que se pueda originar la conformación de unidades económicas dirigidas a satisfacer las necesidades de consumo local.

Por último, cabe mencionar que los esfuerzos realizados por el Estado colombiano para fomentar la asociatividad en el país han sido insuficientes. Aun cuando el marco normativo se ha ajustado para acoger diversas modalidades de las organizaciones de economía solidaria (Ley 79 de 1988 y Ley 454 de 1998), la institucionalidad diseñada para promoverlas ha experimentado un desmonte progresivo. En 2003, mediante Decreto 1799, se restructuró el Departamento Nacional de la Economía Solidaria (Dansocial) creado en 1998 para liderar la política de desarrollo y fortalecimiento de dicho tipo de organizaciones; y en 2011 el Decreto 4122 lo redujo a una Unidad Administrativa Especial de Organizaciones Solidarias (Uaeos), adscrita al Ministerio de Trabajo (Álvarez, 2017).

De acuerdo con un balance sobre la gestión de fomento de la Uaeos, estructurado a partir de encuestas diligenciadas por organizaciones solidarias que han sido asistidas por dicha entidad, se reconoce la cultura de informalidad que frustra los procesos requeridos no solo para la creación de las organizaciones sino para sortear el control al que están sujetas a lo largo de su vida jurídica-como una de las debilidades apremiantes de la asociatividad en el campo. La bancarización, el registro en Cámara de Comercio, los trámites tributarios y la validación periódica de estados financieros son algunos ejemplos. Asimismo, se evidencia la necesidad de fortalecer las estrategias de acompañamiento para que las organizaciones solidarias logren mejorar sus instrumentos productivos, empresariales y modelos de gobernanza para con ello prolongar su permanencia en el mercado (Uaeos, 2015).

\section{Conclusiones}

La historia del pensamiento económico está colmada de ejemplos sobre los fracasos sobrevinientes a la inducción de modelos de desarrollo ajenos a las condiciones culturales, sociales y políticas del entorno en el cual se implementan. Los esquemas asociativos de base comunitaria podrían correr con la misma suerte si se toman como canon para orientar la sustitución de intervenciones públicas en el ámbito del acceso a internet, al amparo de un espejismo que promete resolver problemáticas de sostenibilidad.

En el país, las secuelas de la exclusión social que ha prevalecido en detrimento de la población rural generaron un profundo deterioro del tejido social, se antepuso necesidades de supervivencia al afianzamiento de lazos de confianza, reciprocidad y creación de dinámicas de emprendimiento cooperativo. Bajo estas circunstancias, tal como lo confirma la investigación realizada, no es factible esperar que emerjan espontáneamente iniciativas de base comunitaria para apalancar el despliegue de infraestructura y suplir las necesidades de inclusión digital, sin que antes medie un proceso de cambio estructural.

Descartada esta alternativa, se mantiene abierto el interrogante sobre el devenir de la política de acceso universal. Las preocupaciones acerca de la sostenibilidad y eficiencia de la inversión pública son legítimas cuando se pretende optimizar la asignación de recursos escasos para resolver necesidades latentes y evitar que los beneficiarios de la acción estatal estén supeditados de manera indefinida, a los ciclos de la oferta pública. Sin embargo, en ausencia de una acotación clara de ambos términos -sostenibilidad y eficiencia- en los manifiestos de política pública, cualquier opción que se traduzca en ahorro se podría tomar por sustituto de la acción estatal, sin la rigurosidad que debe ceñir a las decisiones de índole distributiva.

Por lo anterior, cuestionamientos tales como qué beneficios se espera obtener de la difusión tecnológica en las zonas rurales, qué acciones se deben emprender para posibilitar el desarrollo continuo de dicho proceso, o qué efectos podría conllevar para la población rural la inacción pública en materia de acceso a internet, anteceden la 
discusión sobre quién o mediante qué mecanismos se podría financiar el despliegue de infraestructura de telecomunicaciones. Después de todo, entre las orillas del asistencialismo y la autogestión comunitaria hay un vasto entramado de piezas que le dan forma y consistencia al diseño institucional, y que encauzan e instrumentan las aspiraciones de bienestar social.

\section{Referencias}

Alderete, F., y Carrasco, D. (2010). Modelos cooperativos para el acceso a Internet en sectores rurales. La experiencia de Coopesic y sus aprendizajes. Fundación para la Innovación Agraria. http://wwww.redmicrofinanzas.cl/web/wp-content/uploads/2010/07/ Modelos-Cooperativos-USACH.pdf

Álvarez, J. (2017, septiembre). Economía social y solidaria en el territorio: significantes y co-construcción de politicas públicas. Universidad Pontificia Javeriana. http://wwww.javeriana.edu.co/documents/16113/9477233/+2+Econom\%C3\%ADa+social+y+solidaria+en+el+territorio+\%28WEB\%29. pdf/c5355176-c247-4a91-99d3-2a7748164495

Baca, C., Belli, L., Huerta, E., y Velasco, K. (2018). Community Networks in Latin America: Challenges, Regulations and Solutions. Internet Society. https://www.internetsociety.org/wp-content/ uploads/2018/12/2018-Community-Networks-in-LAC-EN.pdf

Banco Mundial. (2001). World Development Report 2000/2001: Attacking Poverty focused on empowerment as a key priority of development policy. https://siteresources.worldbank.org/INTPOVERTY/ Resources/WDR/approutl.pdf

Bardhan, P., y Ray, I. (2008). The Contested Commons: Conversations between Economists and Anthropologists. Blackwell Publishing.
Bennett, S., y D'Onofrio, A. (2014). Beyond critique: Revised approaches to community-driven development. An inception paper. International Rescue Committee. https://assets.publishing.service.gov. uk/media/57a089ae40f0b652dd00035c/61244-Beyond_Critique_Inception_Paper.pdf

Departamento Administrativo Nacional de Estadística (DANE). (2016). Zer Censo nacional agropecuario. Hay campo para todos. https://www.dane.gov. co/files/images/foros/foro-de-entrega-de-resultados-y-cierre-3-censo-nacional-agropecuario/ CNATomoz-Resultados.pdf

Departamento Administrativo Nacional de Estadística (DANE). (2018, abril 9). Boletín técnico Indicadores básicos de tenencia y uso de Tecnologías de la Información y Comunicación en hogares y personas de 5 y más años de edad 2017. http://wwuw. dane.gov.co/files/investigaciones/boletines/tic/ bol_tic_hogares_2017.pdf

Departamento Nacional de Planeación (DNP). (2015, diciembre). Evaluación de Impacto de las iniciativas Kioscos (KVD) y Puntos (PVD) del Plan Vive Digital, asi como del acompañamiento a beneficiarios de la iniciativa Hogares Digitales. Producto 4: Informe de resultados de la evaluación. https://colaboracion.dnp.gov.co/CDT/ Sinergia/Documentos/140_InformeFinal.pdf

Departamento Nacional de Planeación (DNP). (2017, septiembre). Esquema de financiación para el sector TIC y audiovisual en el marco de la convergencia tecnológica y de mercados. https://colaboracion.dnp. gov.co/CDT/Prensa/Publicaciones/20171006\%20 -\%20Esquema\%20de\%20financiaci\%C3\%B3n\%20 TIC\%20vpublicaci\%C3\%B3n.pdf

Fraser, H. (2005). Four different approaches to community participation. Community Development Journal, 40(3), 286-300. https://doi.org/10.1093/cdj/bsi037 
International Telecommunication Union (ITU). (2019). The State of Broadband: Broadband as a Foundation for Sustainable Development. https://wwww.itu.int/ pub/S-POL-BROADBAND.20-2019

Kluz, M. (2017, enero). Cooperatives and Rural Broadband: A Selective Survey. University of Wisconsin. https://resources.uwcc.wisc.edu/Utilities/ CooperativesandBroadbandSurveyz017.pdf

Landriscini, G. (2013). Economía social y solidaria en la Patagonia norte: experiencias, saberes y prácticas. casos y reflexiones. Revista Pilquen, 16(2), 1-15. http://wwww.scielo.org.ar/scielo.php?script=sci_arttext\&pid=\$1851-31232013000200005

Ministerio de Educación Nacional (MEN). (2019). Indicadores de conectividad escolar. Programa Conexión Total, MEN.

Ministerio de Tecnologías de la Información y las Comunicaciones (MinTIC). (2016). Diagnóstico Semestral del Universo de Concesionarios de Radiodifusión Sonora. http://colombiatic.mintic. gov.co/679/articles-73951_recurso_1.pdf

Nguyen, T., y Rieger, M. (2014). Community-driven Development and Social Capital: Evidence from Morocco. EUI Working Paper MUN 2014/02. European University Institute http://cadmus.evi.eu/ bitstream/handle/1814/31037/MWP_2014_02.pdf

Ocampo, J. (2014). Misión para la transformación del campo. Saldar la deuda histórica con el campo. Marco conceptual de la Misión para la Transformación del Campo. Departamento Nacional de Planeación (DNP). https://colaboracion.dnp.gov.co/CDT/Prensa/DOCUMENTO\%20 MARCO-MISION.pdf
Ostrom, E. (1994, junio 2). Neither market nor state: governance of common-poole resources in the twenty-first century. International Food Policy Research Institute, Indiana University https://monoskop.org/ images/d/d8/Ostrom_Elinor_Neither_Market_Nor_ State_Governance_of_Common-Pool_Resouces_ in_the_21st_Century.pdf

Ó Siochrú, S., y Girard, B. (2005). Community-based Networks and Innovative Technologies: New models to serve and empower the poor. United Nations Development Programme Report. http://www.digitalfutures.ca/wp-content/ uploads/2013/10/community-based_nets.pdf

Parsons, S., y Stegeman, J. (2018, julio 11). A Review of Rural Subsidies. Rural Broadband Economics. The Broadband Association. https://www. ustelecom.org/wp-content/uploads/2018/11/ Rural-Broadband-Economics-A-Review-of-RuralSubsidies-final-paper-1.pdf

Programa de las Naciones Unidas para el Desarrollo (PNUD). (2011). Colombia rural: razones para la esperanza. Resumen ejecutivo. Informe Nacional de Desarrollo Humano. PNUD. http://wwww.co.undp.org/content/ dam/colombia/docs/DesarrolloHumano/undp-co-resumen_ejecutivo_indh2011-2011.pdf

Sistema Electrónico para la Contratación Pública (SECOP). (2013). Proceso Licitatorio FTIC-LP-08-2013. https:// www.contratos.gov.co/consultas/detalleProceso. do?numConstancia=13-1-101620

Unidad Administrativa Especial de Organizaciones Solidarias (Uaeos). (2015). Marco para el fomento de la economía solidaria en territorios rurales de Colombia. Unidad Administrativa Especial de Organizaciones Solidarias y Ciriec Colombia. http:// 
www.orgsolidarias.gov.co/sites/default/files/ archivos/Marco\%20de\%20fomento\%20de\%20 la\%20economia\%20solidaria\%20en\%20territorios\%20rurales.pdf

Wong, S. (2012). What have been the Impacts of Bank Community-Driven Development
Programs. COO Impact Evaluation Review and Operational and Research Implications. World Bank. http://documents.worldbank.org/curated/ en/967431468161087566/What-have-been-theimpacts-of-World-Bank-Community-DrivenDevelopment-Programs-CDD-impact-evaluationreview-and-operational-and-research-implications 